\title{
Erratum to: File Type Identification for Digital Forensics
}

\author{
Konstantinos Karampidis ${ }^{(\bowtie)}$ and Giorgios Papadourakis \\ Department of Informatics Engineering, \\ Technological Educational Institute of Crete, Heraklion, Crete, Greece \\ karampidis@outlook.com, papadour@cs.teicrete.gr
}

\section{Erratum to:}

\section{Chapter 25: J. Krogstie et al. (Eds.) Advanced Information Systems Engineering Workshops DOI: 10.1007/978-3-319-39564-7_25}

The authorship of this chapter was incorrectly stated in the chapter and in the index. The names of the authors is reversed.

The authors are Mr. Konstantinos Karampidis and Mr. Giorgios Papadourakis, Department of Informatics Engineering, Technological Educational Institute of Crete, Heraklion, Crete, Greece. 